\section{Investigación en pregrado de las escuelas de medicina de Chile: Motivación y participación de estudiantes de medicina asistentes al Congreso Chileno de Estudiantes de Medicina (COCEM)}

\author{
NICOLÁS SARZOSA ${ }^{1, \mathrm{a}}$, PABLO ARAYA ${ }^{1, \mathrm{a}}$, MARCO RUIZ ${ }^{1, \mathrm{a}}$, \\ MELISSA ARAYA ${ }^{1, a}$, CATALINA BIÉNZOBAS $^{1, \mathrm{a}}$, \\ SLAVKA CHELEBIFSKI I,a, MARÍA JOSÉ AYALA ${ }^{1, \mathrm{a}}$, \\ CLAUDIA G. SÁEZ ${ }^{1}$
}

\section{Participation of Chilean undergraduate medical students in scientific research}

Background: The participation of medical students in research generates professional, scientific, and personal benefits for the student. Aim: To evaluate the interest and opportunities for medical students in Chile to participate in scientific research and their perceptions about factors influencing research. Material and Methods: All students attending the 2018 Chilean Congress for Medical Students were invited to answer a 44 questions survey about interest and opportunities to participate in research. Results: The survey was answered by 489 of the 538 students attending the congress. Eighty five percent referred interest in conducting scientific research, but only $47 \%$ had the opportunity to actively participate in a research project. The main research area providing opportunities was epidemiology and the main form to access a research project was through direct contact with a medical professor or researcher. Seventy seven percent of respondents had courses of scientific investigation in their medical curriculum and $92 \%$ had a scientific society for medical students in their university. Conclusions: Respondents showed a great deal of interest in participating in scientific research. However, there is a gap between this interest and the available opportunities. Medical professors should promote and facilitate the participation of their students in research.

(Rev Med Chile 2020; 148: 1825-1832)

Key words: Education, Medical; Education, Medical, Undergraduate; Research; Students, Medical.
${ }^{1}$ Escuela de Medicina, Pontificia Universidad Católica de Chile.

Santiago, Chile.

aEstudiante de Medicina.

Financiamiento: este estudio recibió aportes de la

Dirección de Pregrado de

Medicina de la Pontificia

Universidad Católica de Chile

para la impresión de los

documentos empleados para el

estudio y La Academia Científica

de Estudiantes de Medicina de la

Pontificia Universidad Católica de

Chile (ACEM UC).

Los autores declaran no tener

conflictos de interés.

Recibido el 13 de mayo de 2020, aceptado el 26 de octubre de 2020.

\section{Correspondencia a:}

Nicolás Sarzosa Adaos

Diagonal Paraguay 362, primer piso. Santiago, Chile.

nisarzosa@uc.cl

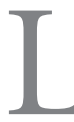

a producción de nuevo conocimiento científico es fundamental en el desarrollo de las sociedades. En salud, para que logre ser un beneficio es esencial que los médicos puedan plantear y desarrollar dudas clínicamente relevantes $^{1,2}$. En este sentido, el aprendizaje científico es fundamental para la formación médica y el continuo progreso del conocimiento biomédico, al producir nuevos investigadores ${ }^{2,3}$.

En los últimos años, Chile ha disminuido el porcentaje de médicos responsables de investigaciones en el Fondo Nacional de Investigación 
en Salud ${ }^{4}$, atribuido posiblemente a la escasa experiencia, visión o preparación investigativa a nivel de pregrado 5 . Asimismo, está descrito que la formación en competencias de investigación en programas de postítulo de médicos especialistas no es suficiente para mantener un índice de producción científica adecuada de acuerdo a ISI (actualmente $\mathrm{WoS})^{6}$.

La participación de parte de los estudiantes de medicina en investigación está asociada a más producción científica y a mayor éxito en seguir una carrera de investigador ${ }^{7}$. Se ha reportado que realizar investigación en pregrado influye en la contratación docente, promoción laboral, salario, reputación y a la competitividad del estudiante para elegir trabajo o residencia ${ }^{8}$. Un estudio cualitativo reportó que los estudiantes involucrados en investigación en pregrado tuvieron mejor aproximación a los conceptos de autonomía, colaboración entre pares y construcción del conocimiento desde distintas perspectivas ${ }^{9}$. En consecuencia, la investigación en pregrado trae beneficios al generar competencias que permiten el desarrollo profesional, abriendo incluso posibilidades en la educación continua.

En Chile no existen estudios formales que evalúen el estado de interés y participación de los estudiantes de pregrado de medicina en investigación. Se han hecho acercamientos, como una carta al editor, presentada por Clouet-Huerta et al. en la Revista Médica de Chile, donde presentan una reducción anual del número de trabajos presentados en la competencia científica del Congreso Científico Nacional de Estudiantes de Medicina (CCNEM) durante el período 2005-2013, resultados discutidos en cartas al editor posteriores ${ }^{10,11,12}$. Por su parte, Gutiérrez et al. analizan la cantidad de trabajos presentados en el periodo 2006-2014, tanto de las distintas versiones del CCNEM como del Congreso Científico Temático de Estudiantes de Medicina (CCTEM), llegando a conclusiones similares ${ }^{13}$. A nivel internacional, Mayta-Tristán et al. evaluaron las habilidades de investigación en estudiantes de pregrado de medicina latinoamericanos, donde estudiantes chilenos declararon tener un mayor nivel en búsqueda de información, lectura crítica y proceso de publicación ${ }^{14}$.

Durante los últimos años, las academias científicas de estudiantes de medicina de la Pontificia Universidad Católica de Chile y de la Universidad de Chile han organizado el Congreso Chileno de
Estudiantes de Medicina (COCEM), instancia que reúne alrededor de 500 estudiantes de medicina de todo Chile. En este evento se realizan charlas expositivas, talleres prácticos y una competencia científica.

En el contexto que el congreso ofrece, se planteó como objetivo de este estudio conocer el estado de investigación en pregrado de las escuelas de medicina de Chile, referido por los asistentes al III COCEM del año 2018. Se evaluó el interés de los estudiantes por investigar y su grado de participación, el entorno institucional de investigación, así como percepciones sobre factores que influyen en investigar.

\section{Materiales y Métodos}

Este es un estudio individual, transversal, analítico. Se aplicó una encuesta a estudiantes de medicina chilenos, asistentes al III COCEM realizado en Santiago de Chile, durante los días 27 y 28 de julio de 2018.

Se incluyeron en el estudio estudiantes de pregrado de la carrera de medicina, pertenecientes a universidades chilenas acreditadas, y que firmaron el consentimiento informado aprobado por el comité de ética de la Pontificia Universidad Católica de Chile ( $\mathrm{n}^{\circ}$ proyecto: 190314003 ).

La encuesta fue confeccionada por el equipo investigador (autores), con el objetivo de evaluar el interés y participación de estudiantes de pregrado de medicina en investigaciones, así como el entorno de investigación y factores que influyen en realizar esta. El instrumento consistió en una encuesta anónima impresa de 44 preguntas (adjunta en Anexo 1). Esta incluyó 5 secciones: datos demográficos ( 6 preguntas), interés y grado de participación en investigación (9 preguntas), entorno de investigación (malla curricular y sociedades científicas de estudiantes de medicina, 6 preguntas), facilitadores y limitaciones para investigar ( 1 pregunta abierta) y percepciones sobre factores que influyen en investigación (curriculum, docentes, tiempo, motivación personal, entre otros; 22 preguntas) evaluadas a través de la escala de Likert ("Muy en desacuerdo", "En desacuerdo", "Me es indiferente", "De acuerdo", "Muy de acuerdo" y "Desconozco"). En la sección de interés y grado de participación en investigación, los estudiantes tuvieron la oportunidad de reportar la cantidad de investi- 
gaciones en las que habían participado y clasificarlas. Respecto a la clasificación, se tomaron en consideración las respuestas de quienes habían realizado sólo una investigación. No se analizó la pregunta abierta de facilitadores y limitaciones en el presente estudio.

El estudio fue complementado con información de sitios web oficial sobre años de acreditación de las universidades de origen de los participantes. Además, se realizó una búsqueda sobre la presencia de cursos de investigación en cada malla curricular y de academias o sociedades científicas de estudiantes de medicina, a través de los respectivos sitios web y redes sociales de cada facultad.

Los resultados se presentan como número de casos y porcentajes. Se utilizó el test chi-cuadrado de Pearson para la asociación de variables categóricas. Se usó razón de chances (OR) para determinar la chance de hacer investigación de un grupo de alumnos respecto a otro.

Se usó un nivel de significancia de 5\%, para todas las comparaciones. La información extraída fue digitalizada utilizando el programa Microsoft Excel $^{\circ}$. Los datos se analizaron con el programa IBM SPSS Statistics $25^{\circ}$.

\section{Resultados}

\section{Datos demográficos}

Este estudio incluyó 489 participantes, provenientes de todas las regiones del país y 24 universidades nacionales. El 54,40\% de la población era femenina y el promedio de edad correspondió a 21,83 $\pm 2,21$ años (rango: 18-38 años). En la Tabla 1 se detalla: región de residencia y estudio, año de carrera según avance curricular y años de acreditación. El 82,4\% de los participantes estudiaba en la misma región donde residía. El 51,74\% de los participantes residía en la Región Metropolitana y $57,06 \%$ estudiaba en esta misma región. Adicionalmente, $64,42 \%$ estudiaba en escuelas de medicina con más de 5 años de acreditación.

\section{Interés y grado de participación en investigación}

Resultados se resumen en Tabla 2. El 84,84\% de los participantes afirmó haber estado interesado en realizar una investigación durante la carrera, pero de estos, solo $46,62 \%$ tuvo la oportunidad de investigar. De los que estudiaban fuera de la Región Metropolitana (RM), 37,1\% tuvo oportu-

Tabla 1. Características demográficas de la población

\begin{tabular}{|lcc|}
\hline Característica & $\mathbf{n}=\mathbf{4 8 9}$ & (\%) \\
Género femenino & 266 & $54,40 \%$ \\
Edad & $21,83 \pm 2,21$ años & - \\
Región de residencia (RM) & 253 & $51,74 \%$ \\
Región donde estudia (RM) & 279 & $57,06 \%$ \\
\hline Curso & & $7,98 \%$ \\
Primer año & 39 & $23,11 \%$ \\
Segundo año & 113 & $21,88 \%$ \\
Tercer año & 107 & $23,31 \%$ \\
Cuarto año & 114 & $21,68 \%$ \\
Quinto año & 106 & $0,41 \%$ \\
Sexto año & 2 & $1,64 \%$ \\
Séptimo año & 8 & $0,20 \%$ \\
Años de acreditación de la Universidad & & $5,52 \%$ \\
Dos años & 1 & $3,68 \%$ \\
Tres años & 27 & $26,17 \%$ \\
Cuatro años & 18 & $32,51 \%$ \\
Cinco años & 128 & $31,69 \%$ \\
Seis años & 159 & \\
Siete años & 155 & \\
\hline
\end{tabular}


Tabla 2. Interés, grado de participación y entorno de investigación

\begin{tabular}{|c|c|c|}
\hline Característica & $\mathbf{n}$ & $\%$ \\
\hline Interés en investigar ( $\mathrm{n}=488$ ) (Sí) & 414 & 84,84 \\
\hline Oportunidad de investigar ( $n=488$ ) (Sí) & 208 & 42,54 \\
\hline Número de investigaciones por estudiante $(n=194)$ & $1,40 \pm 0,84$ & - \\
\hline \multicolumn{3}{|l|}{ Área de investigación ( $n=132$ ) } \\
\hline Ciencias básicas & 31 & 23,48 \\
\hline Epidemiología & 53 & 40,15 \\
\hline Estudios clínicos & 33 & 25,00 \\
\hline Ética & 3 & 2,27 \\
\hline Otros & 12 & 9,09 \\
\hline \multicolumn{3}{|l|}{ Modos de acceso a investigación reportados $(n=211)$} \\
\hline "Yo lo propuse" & 39 & 14,22 \\
\hline "El doctor lo propuso en su clase/rotación" & 63 & 29,86 \\
\hline "Contacté a un doctor, con el interés de participar" & 62 & 29,38 \\
\hline "Me llegó una invitación vía correo institucional" & 17 & 8,06 \\
\hline Otros & 39 & 18,48 \\
\hline \multicolumn{3}{|l|}{ Rol en la investigación ( $n=194)$} \\
\hline Diseño & 42 & 21,65 \\
\hline Recolección de información & 110 & 56,70 \\
\hline Tabular y/o analizar datos & 113 & 58,25 \\
\hline Redacción del informe & 71 & 36,60 \\
\hline Otros & 13 & 6,70 \\
\hline \multicolumn{3}{|l|}{ Motivo de finalización del proyecto $(n=131)$} \\
\hline El proyecto finalizó con éxito & 63 & 48,09 \\
\hline El proyecto no prosperó & 11 & 8,40 \\
\hline El proyecto está congelado & 21 & 16,03 \\
\hline Renuncié al proyecto & 14 & 10,69 \\
\hline Me pidieron que me retirara & 2 & 1,53 \\
\hline Otra & 20 & 15,27 \\
\hline \multicolumn{3}{|l|}{ Influencia del ramo en la motivación $(n=303)$} \\
\hline "Me motivó aún más en mi presente por investigar" & 81 & 26,64 \\
\hline "Me motivó en mi entonces ausente interés por investigar" & 61 & 20,07 \\
\hline "No influyó en mi entonces presente interés" & 87 & 28,62 \\
\hline "No influyó en mi ausente interés por investigar" & 21 & 6,91 \\
\hline "Me desmotivó en mi entonces presente interés" & 35 & 11,51 \\
\hline "Me desmotivó más en mi ausente interés por investigar" & 7 & 2,31 \\
\hline "Otro" & 11 & 3,63 \\
\hline \multicolumn{3}{|l|}{ Entorno de Investigación } \\
\hline Participantes cuya malla curricular tiene ramos de investigación $(n=488)$ & 375 & $76,8 \%$ \\
\hline $\begin{array}{l}\text { Participantes cuya carrera tiene alguna organización científica de estudiantes de } \\
\text { medicina }(n=488)\end{array}$ & 450 & $92,2 \%$ \\
\hline Participantes de alguna organización científica de estudiantes de medicina $(n=475)$ & 50 & $10,5 \%$ \\
\hline
\end{tabular}

Se describen los resultados en torno a interés en investigación, definida como la cantidad de encuestados que declaró haber tenido o tener interés en investigar. A su vez, se describen las respuestas de aquellos encuestados que refirieron haber participado en proyectos de investigación y cuáles eran las características del entorno de aquel proyecto. A su vez, se describen la presencia o ausencia de ramos de investigación y entorno relacionado a investigación de cada estudiante. 
nidad para investigar, comparado con $46,7 \%$ de estudiantes de la RM $(\mathrm{p}=0,033)$. Al comparar por región de residencia, $83,4 \%$ de los residentes fuera de la RM tuvo interés en investigar y, de estos, el $40,3 \%$ tuvo la oportunidad para hacerlo. Por otro lado, $86,2 \%$ de los residentes en la RM presentó interés $(p=0,395)$, pero solo $52,5 \%$ tuvo oportunidad ( $\mathrm{p}=0,014, \mathrm{OR}=0,64$, IC95\%: 0,44-0,91).

De los interesados por investigar, $30,1 \%$ correspondía a primer y segundo año de carrera y de estos, 25,6\% refirió haber tenido oportunidad para investigar, mientras que $69,9 \%$ eran de cursos superiores y $55,7 \%$ de ellos refirió haber tenido una oportunidad. Tener interés por investigar y pertenecer a cursos superiores se asoció a cerca de 2,2 veces más probabilidad de investigar, respecto a quienes pertenecían a primer y segundo año $(\mathrm{p}<0,001, \mathrm{OR}=2,17, \mathrm{IC} 95 \%=1,46-3,21)$.

\section{A. Investigación realizada}

Del total de personas que reconocieron haber tenido la oportunidad de investigar, el $64,73 \%$ participó en 1 proyecto, $21,26 \%$ en 2 y $7,25 \%$ en 3 o más. El 5,8\% no respondió.

El área de investigación más reportada significativamente fue epidemiología $(\mathrm{p}<0,005)$, seguida por estudios clínicos y ciencias básicas.

Los estudiantes que han participado en investigación reportaron dos principales formas para incorporarse a un estudio: aceptar la invitación de un docente en su clase o rotación $(29,86 \%)$ o contactar directamente a un docente por iniciativa del alumno (29,38\%). Los estudiantes reportaron desempeñarse principalmente en recolección de información y en tabulación y análisis de datos. Respecto a la cantidad de tareas llevadas a cabo, $57,22 \%$ de los estudiantes se desenvolvió en uno de los roles, $20,10 \%$ se dedicó a dos, 9,28\% a tres y $13,40 \%$ a cuatro o más.

\section{B. Percepción de trascendencia de la investigación realizada}

Los participantes evaluaron del 1 al 10 el nivel de impacto o relevancia que percibían de su investigación. De las 201 investigaciones reportadas, $42,79 \%$ calificó su investigación de 4 a $6,35,82 \%$ de 7 a $9,15,42 \%$ de 1 a 3 , y solo $5,97 \%$ con la calificación máxima de 10 .

Respecto a la finalización de la investigación, $48,09 \%$ de la muestra afirmó haber finalizado el proyecto con éxito, $16,03 \%$ refirió proyecto con- gelado y $10,69 \%$ renunció por causas no especificadas. El 8,40\% de los estudiantes que investigaron declaró que el proyecto no prosperó.

\section{Entorno institucional de investigación}

En cuanto a malla curricular, $76,8 \%$ de los participantes estudiaba en una escuela de medicina cuya malla incluye cursos de investigación (Tabla 2). Al comparar las respuestas de los participantes, $26,4 \%$ desconocía si su malla curricular presenta o no estos cursos como parte del currículum obligatorio. Ante la pregunta de cómo influyó dicho curso, 46,71\% de los estudiantes manifestó motivarse por investigar debido al ramo, y de estos, aproximadamente la mitad se motivó, sin estarlo previamente.

Según la información disponible, 92,2\% de los encuestados estudiaba en una facultad que presenta academias o sociedades científicas de estudiantes de medicina. Pese a esto, $28,4 \%$ de los participantes desconocía que su carrera presentara estas academias. Del total de aquellos que afirmaron tener organizaciones estudiantiles en su universidad, 10,5\% afirmó participar activamente.

\section{Percepciones sobre factores que influyen en investigación}

Con el objetivo de evaluar las opiniones de los participantes acerca de distintos factores que influyen en investigar, se realizó una encuesta con respuestas según escala Likert. Los resultados se resumen en la Figura 1.

El 90,59\% de los participantes estuvo de acuerdo con el enunciado "El desarrollo de nuevo conocimiento en biomedicina es fundamental para mi formación médica”. El 84,66\% estuvo de acuerdo con "La falta de tiempo por mi carga académica ha limitado mi posibilidad de poder participar en un equipo investigador", mientras que $60,57 \%$ reportó falta de tiempo independiente de su carga académica. El 82,79\% señaló que se sumaría a un equipo de investigación si dispusiese de más tiempo.

El 66,60\% de los estudiantes manifestó estar muy de acuerdo/de acuerdo con la afirmación "en mi universidad se realizan proyectos de investigación de calidad, que resultan en un aporte sustancial a Chile y la comunidad científica". Estudiar en una universidad fuera de la RM se encontró asociada a una menor probabilidad de estar 


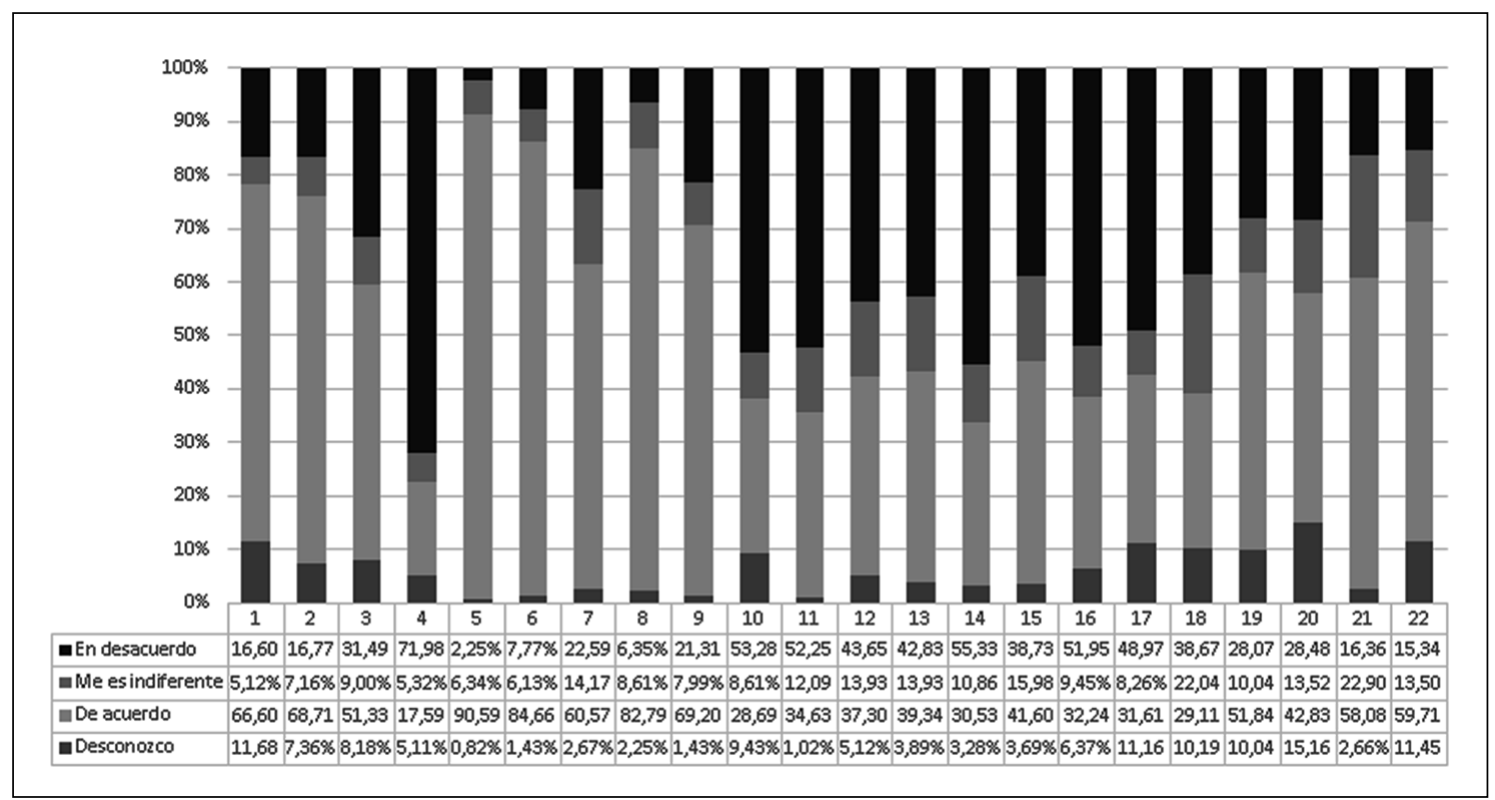

Figura 1. Percepción sobre factores que influyen en investigar. 1: En mi universidad se realizan proyectos de investigación de calidad, que resultan en un aporte sustancial a Chile y la comunidad científica. 2: El curriculum de mi facultad tiene entre sus objetivos lograr que desarrollemos habilidades básicas de investigación. 3: Los docentes de mi facultad invitan a alumnos de pregrado a realizar una inmersión en su inmersión en su laboratorio o a trabajar en conjunto en uno de sus proyectos de investigación vigente. 4: En mi horario habitual existe un espacio de tiempo que puede utilizarse para realizar actividades de investigación. 5: El desarrollo de nuevo conocimiento en biomedicina es fundamental para mi formación médica. 6: La falta de tiempo por mi carga académica ha limitado mi posibilidad de poder participar en un equipo investigador. 7: La falta de tiempo independiente de mi carga académica ha limitado mi posibilidad de poder participar en un equipo investigador. 8: Si dispusiese de más tiempo, me sumaría a un equipo de investigación. 9: La falta de información o cómo acceder a ésta ha limitado mi posibilidad de poder participar en un equipo de investigación. 10: Conozco los programas de investigación que ofrece mi escuela. 11: La falta de motivación personal ha limitado mi posibilidad de poder participar en un equipo investigador. 12: La falta de capacidades técnicas han limitado mi posibilidad de poder participar en un equipo investigador. 13: La falta de capacidades en estadística han limitado mi posibilidad de poder participar en un equipo investigador. 14: La falta de capacidades en metodología de la investigación ha limitado mi posibilidad de poder participar en un equipo investigador. 15: La falta de conocimiento en la línea de investigación que me interesa han limitado mi posibilidad de poder participar en un equipo investigador. 16: La falta de infraestructura en mi facultad han limitado mi posibilidad de poder participar en un equipo investigador. 17: La falta de académicos/as investigadores disponibles en mi facultad han limitado mi posibilidad de poder participar en un equipo investigador. 18: El testimonio de académicos/as investigadores sobre la dificultad en la obtención de fondos ha limitado mi interés por participar en un equipo investigador. 19: Las organizaciones estudiantiles (ACEM, centros de estudiantes, etc.) juegan un rol fundamental a la hora de difundir sobre los programas disponibles para investigar en mi facultad. 20: Las organizaciones estudiantiles (ACEM, centros de estudiantes, etc.) juegan un rol fundamental a la hora de capacitarse para participar de un equipo de investigación en mi facultad. 21: La competencia científica de los congresos estudiantiles corresponden a una instancia de gran interés para mí. 22: La competencia científica de los congresos estudiantiles sirven fundamentalmente para la obtención de puntaje en el concurso de becas del Minsal.

de acuerdo/muy de acuerdo con esta afirmación (78,5\% de RM vs 50,7\% otra región, $\mathrm{p}<0,001)$.

Respecto a la afirmación "La falta de académicos/as investigadores disponibles en mi facultad han limitado mi posibilidad de poder participar en un equipo investigador", $31,61 \%$ estuvo de acuerdo/muy de acuerdo. Estudiar en una universidad fuera de la RM se asoció a mayor probabilidad de estar de acuerdo/muy de acuerdo con esta afirmación $(\mathrm{p}<0,001)$.

\section{Discusión}

En 2018 asistieron cerca de 500 estudiantes de medicina a COCEM, de los cuales, aproximadamente, 93\% participó en este estudio, prove- 
nientes de 24 universidades, con una proporción de género equitativa. Comparativamente, en un estudio previo, se incluyeron 208 estudiantes (101 chilenos), de ocho países de América Latina y 35 universidades, con el objetivo de identificar la apreciación de estos sobre el nivel de capacitación que las universidades aportan en investigación, concluyendo que los procesos de investigación y publicación son reportados como deficientes ${ }^{14}$. Nuestro estudio incluye mayor cantidad de participantes y evalúa la realidad chilena respecto a: interés y oportunidad de investigar, rol desempeñado por el alumno en investigación y percepciones sobre la investigación.

COCEM es un congreso científico, por ende, quienes asisten tienen un probable mayor interés en investigación o en adquirir nuevo conocimiento biomédico, además de poseer recursos económicos y tiempo para asistir. Los asistentes a COCEM-2018 representan 3,2\% de los estudiantes de medicina chilenos ${ }^{15}$, pese a que el grupo seleccionado tiene un interés especial por investigación, destaca que solo $46 \%$ ha podido incorporarse a estudios de investigación, lo que demuestra una brecha entre mayor interés de investigar y menor oportunidad para realizarlo. Similarmente, un estudio brasileño incluyó 415 estudiantes que cursaban la carrera de medicina durante 2009, seleccionados sin considerar involucramiento en investigación, reportando que 52,8\% de ellos no participó en proyectos de investigación, pero $85,4 \%$ mostró interés en investigar ${ }^{16}$.

Varios reportes han descrito al factor tiempo como una de las principales limitaciones para investigar ${ }^{17-20}$, lo que coincide con nuestros hallazgos. Los participantes reportan la falta de tiempo como limitación, ya sea por carga académica como independientemente de esta. Cabe destacar que los estudiantes del estudio refirieron estar dispuestos a participar de una investigación si su percepción de disponibilidad de tiempo mejorase.

Otra limitación observada es estudiar en regiones, puesto que $46,7 \%$ de los estudiantes de la RM reporta haber tenido oportunidad de investigar, en contraste con $37,1 \%$ en estudiantes fuera de esta. A su vez, estos últimos se mostraron menos de acuerdo con que en su universidad se realizan proyectos de calidad, y significativamente más de acuerdo con que la falta de docentes ha reducido aun más su posibilidad de participación.

Paralelamente, se observó que estudiantes de cursos superiores presentan significativamente mayor oportunidad de investigar que aquellos de primeros años. Asimismo, la mayoría de los que realizaron investigación lo hicieron gracias a la propuesta o contacto directo con un docente, evidenciando la importancia de los académicos en potenciar la participación de los estudiantes en investigación. Está reportado que los investigadores con grado académico o estudiantes de postdoctorado, están motivados a ser mentores de estudiantes de pregrado para mejorar su productividad científica y desarrollar habilidades docentes ${ }^{21}$, sugiriendo que la brecha entre interés y oportunidad, que evidencia nuestro estudio, podría ser explicada por falta de difusión u organismos que vinculen ambos actores, lo que podrá ser abordado en investigaciones futuras.

Este estudio, realizado por estudiantes de medicina, cuyo objetivo era evaluar y caracterizar la participación de estudiantes de medicina en proyectos científicos, permite concluir que: existe una brecha entre interés y oportunidad para investigar, que el rol del docente es relevante para la incorporación de estudiantes a proyectos científicos y que el tiempo del estudiante es una de las limitaciones más importantes para participar en proyectos. Las variables causantes de estos hallazgos deberán ser abordadas en futuras investigaciones.

Agradecimientos: Agradecemos a la Dirección de Pregrado de Medicina de la Pontificia Universidad Católica de Chile y a la Academia Científica de Estudiantes de Medicina UC por su apoyo para realizar este trabajo. Agradecemos también al Dr. Mauricio Cuello F. por guiarnos en el diseño y redacción de esta investigación y al Dr. Luis Villaroel D. por guiarnos en el diseño estadístico de nuestro estudio.

\section{Referencias}

1. Horton B. From bench to bedside... Research makes the translational transition. Nature 1999; 402 (6758): 213-5.

2. Pober JS, Neuhauser C, Pober J. Obstacles facing translational research in academic medical centers. FASEB J 2001; 15 (13): 2303-13.

3. Enhancing the clinical research pipeline: training approaches for a new century. Acad Med 2001; 76 (4): 307-15.

4. Labbe T, Barake F, Alamos M, Molina C, Rios J. Reali- 
dad de la Investigación en Salud en Chile: participación de los Médicos en el Fondo Nacional de Investigación (FONIS) en la última década. Ars Med 2017; 42 (2): 7680.

5. Salas S, Rigotti R. Médicos Científicos en Chile: ¿Una especie en extinción? Rev Med Chile 2005; 133 (1): 1218.

6. Concha M, Miranda J, Vergara G, Ibarra H. [Commitment and development of competences in clinical research in residency training programs in Chile]. Rev Med Chile 2012; 140 (3): 326-33.

7. Amgad M, Man Kin Tsui M, Liptrott S, Shash E. Medical Student Research: An Integrated Mixed-Methods Systematic Review and Meta-Analysis. plos ONE 2015; 10 (6): 1-31. Disponible en: https://doi.org/10.1371/ journal.pone.0127470 [Consultado el 12 de noviembre de 2019].

8. Ávila M, Rodríguez-Restrepo A. The importance of research in undergraduate medical education. Medwave 2014; 14 (10):1-7. Disponible en: https://www.medwave. cl/link.cgi/Medwave/Revisiones/revisiontemas/6032 [Consultado el 10 de noviembre de 2019].

9. Imafuku R, Saiki T, Kawakami C, Suzuki Y. How do students' perceptions of research and approaches to learning change in undergraduate research? Int J Med Educ 2015; 6: 47-55.

10. Clouet-Huerta D, Correa U. [Undergraduate medical research: what is going on in Chile?]. Rev Med Chile 2014; 142 (11): 1488-90.

11. Araos-Baeriswyl E, Moll-Manzur C. [How many undergraduate medical students are involved in medical research?]. Rev Med Chile 2015; 143 (10): 1358-9.

12. Clouet-Huerta D, Correa U. [How many undergraduate medical students are involved in medical research? A reply]. Rev Med Chile 2015; 143 (10): 1359-60.

13. Gutiérrez D, Cid C. Undergraduate medical research requires change. Medwave 2015; 15 (4). Disponible en:
https://www.medwave.cl/link.cgi/English/Perspectives/ letterstoeditor/6159.act [Consultado el 10 de noviembre de 2019].

14. Mayta-Tristán P, Cartagena-Klein R, Pereyra-Elías R, Portillo A, Rodríguez-Morales A. [Latin American medical students' appraisal on university scientific research training]. Rev Med Chile 2013; 141 (6): 716-22.

15. Base de datos de Matrícula Histórica 2007 - 2019 Ministerio de Educación de Chile. Disponible en: https:// www.mifuturo.cl/bases-de-datos-de-matriculados/ [Consultado el 5 de mayo de 2020].

16. Oliveira C, de Souza R, Abe E, Silva L, de Carvalho $\mathrm{L}$, Domingues M. Undergraduate research in medical education: a descriptive study of students' views. BMC Med Educ 2014; 14 (51): 1-8. Disponible en: https:// doi.org/10.1186/1472-6920-14-51 [Consultado el 4 de septiembre de 2019].

17. Siemens DR, Punnen S, Wong J, Kanji N. A survey on the attitudes towards research in medical school. BMC Med Educ 2010; 10 (4): 1-7. Disponible en: https:// doi.org/10.1186/1472-6920-10-4 [Consultado el 18 de noviembre de 2019].

18. Chang Y, Ramnanan C. A review of literature on medical students and scholarly research: experiences, attitudes, and outcomes. Acad Med 2015; 90 (8): 116273.

19. Hamdan A, Kakarla J. Barriers faced by medical students interested in research. Med Teach 2013;35 (1): 78.

20. Russell C, Lawson A, macgregor K, Millar F, Young A, Funston G. Perceived barriers to research in undergraduate medicine. Med Teach 2012; 34 (9): 777-8.

21. Limeri LB, Asif MZ, Dolan EL. Volunteered or voluntold? The motivations and perceived outcomes of graduate and postdoctoral mentors of undergraduate researchers. CBE- Life Sci Educ 2019; 18 (2): 1-18. Disponible en: https://doi.org/10.1187/cbe.18-10-0219 [Consultado el 20 de septiembre de 2019]. 